\title{
Vertebral osteomyelitis and epidural abscess caused by gas gangrene presenting with complete paraplegia: a case report
}

\author{
Manabu Akagawa ${ }^{1 *}$, Takashi Kobayashi ${ }^{1}$, Naohisa Miyakoshi' ${ }^{2}$ Eiji Abe ${ }^{1}$, Toshiki Abe ${ }^{1}$, Kazuma Kikuchi ${ }^{1}$
} and Yoichi Shimada ${ }^{2}$

\begin{abstract}
Introduction: Gas gangrene is most often caused by Clostridium perfringens infection. Gas gangrene is a medical emergency that develops suddenly. The mortality rate is higher with trunk involvement than with involvement of the extremities, which carries a better prognosis. With respect to vertebral involvement, there are few reports in the literature. The purpose of this paper is to report a very rare case of vertebral osteomyelitis caused by gas gangrene.

Case presentation: A 78-year-old Japanese woman with diabetes mellitus was admitted to our hospital with the chief complaints of back pain, dysuria, and complete paralysis of both legs. A computed tomography scan showed soft tissue swelling anterolaterally at intervertebral disc level T11/12 and a gas-containing epidural abscess that compressed her spinal cord. Cultures later grew Clostridium perfringens and Escherichia coli. Hemilaminectomy was done from T10 to T12, and an epidural abscess was removed. She went on to have fusion surgery 6 weeks after the initial operation and subsequently experienced complete pain relief. She was discharged 2 months later, at which time she was able to walk with a cane. Examination 18 months after surgery showed normal gait without a cane.
\end{abstract}

Conclusions: Discitis caused by gas gangrene infection was successfully treated by immediate debridement and subsequent fusion surgery.

Keywords: Clostridium perfringens, Debridement, Epidural abscess, Fusion surgery, Gas gangrene, Vertebral osteomyelitis

\section{Introduction}

Spontaneous gas gangrene is most often caused by a bacterium called Clostridium perfringens [1]. Gas gangrene is one of the most fulminant infectious diseases; it can cause myonecrosis, gas production, and sepsis. Progression of toxemia and shock is often very rapid. The mortality rate is higher with trunk involvement than with involvement of the extremities, which carries a better prognosis [2]. Antibiotics alone are not effective because they do not penetrate ischemic muscles sufficiently [3]. With respect to vertebral involvement, there are few reports in the literature [4-10]. The purpose of this paper is to report a very rare case of vertebral osteomyelitis caused by gas gangrene.

\footnotetext{
* Correspondence: akagawamanabu@yahoo.co.jp

'Department of Orthopedic Surgery, Akita Kousei Medical Center, 1-1-1 lijima-Nishifukuro, Akita 011-0948, Japan

Full list of author information is available at the end of the article
}

\section{Case presentation}

A 78-year-old Japanese woman with diabetes mellitus was admitted to our institution with a 1-week history of back pain and a 1-day history of paralysis of both legs. She had been bedridden for a week prior to admission in another hospital. There was no history of trauma. No gastrointestinal symptoms were present. Her temperature was $36.8^{\circ} \mathrm{C}$, blood pressure was $100 / 70 \mathrm{mmHg}$, and pulse was 103 beats per minute. On physical examination, her patellar tendon reflexes were present, but Achilles tendon reflexes were absent. All sensory modalities were absent below the inguinal level. Her leg muscles were completely paralyzed bilaterally. There was no anal wink. Anal tone was diminished, and there was no voluntary contraction of the external sphincter.

Admission laboratory values included a white blood cell count (WBC) of 18,800 per $\mathrm{mm}^{3}$, her hemoglobin 
was $9.4 \mathrm{~g} / \mathrm{dL}$, and her hematocrit was $30 \%$. Her Westergren erythrocyte sedimentation rate was $80 \mathrm{~mm} /$ hour, and C-reactive protein (CRP) was $30.02 \mathrm{mg} / \mathrm{dL}$. Initial radiographic examination of her lumbar spine at admission showed only spondylotic change. A computed tomography (CT) scan showed soft tissue swelling anterolaterally at intervertebral disc level $\mathrm{T} 11 / 12$ and a gas-containing epidural abscess that compressed her spinal cord (Figure 1). Magnetic resonance imaging showed involvement of the T11/12 disc space and adjacent vertebral body with decreased signal intensity on T1-weighted images and increased signal intensity on T2-weighted images with a gas-containing epidural abscess (Figure 2).

The patient was urgently admitted to our hospital and taken to the operating room for decompression and disc debridement. Hemilaminectomy was done from T10 to T12, and the epidural abscess was removed, followed by T11/12 disc debridement. The epidural abscess was yellow and turbid. Cultures later grew C. perfringens and Escherichia coli. The wound was closed over subfascial drains.

On the second postoperative day, she was confused, and hydrothorax appeared. Thoracic drainage was then performed by a surgeon. Intravenous antibiotic treatment was begun with imipenem/cilastatin at $0.5 \mathrm{~g}$ every 8 hours for 2 weeks, followed by piperacillin/ tazobactam at $1.0 \mathrm{~g}$ every 8 hours for 2 weeks, and then ampicillin-sulbactam at $1.0 \mathrm{~g}$ every 8 hours for 2 weeks.

Postoperatively, she experienced some sensory and motor return in her legs, but back pain and left chest pain in the sitting position continued. A CT scan 6 weeks after the operation showed T12 vertebral bone destruction (Figure 3); her WBC count and CRP were 8800 per $\mathrm{mm}^{3}$ and $0.26 \mathrm{mg} / \mathrm{dL}$, respectively. She went on to have fusion surgery with instrumentation and subsequently experienced complete relief of her pain. She was discharged 2 months later, at which time she was able to walk with a cane. Examination 18 months after surgery showed normal gait without a cane. Plain radiograph at 18 months after operation showed complete union between the T11 and T12 vertebral bodies (Figure 4).

\section{Discussion}

Gas gangrene is divided into the following three types: posttraumatic, postoperative, and spontaneous [11]. A literature review reported that the spontaneous type accounts for $16 \%$ of cases [11]. Spontaneous clostridial

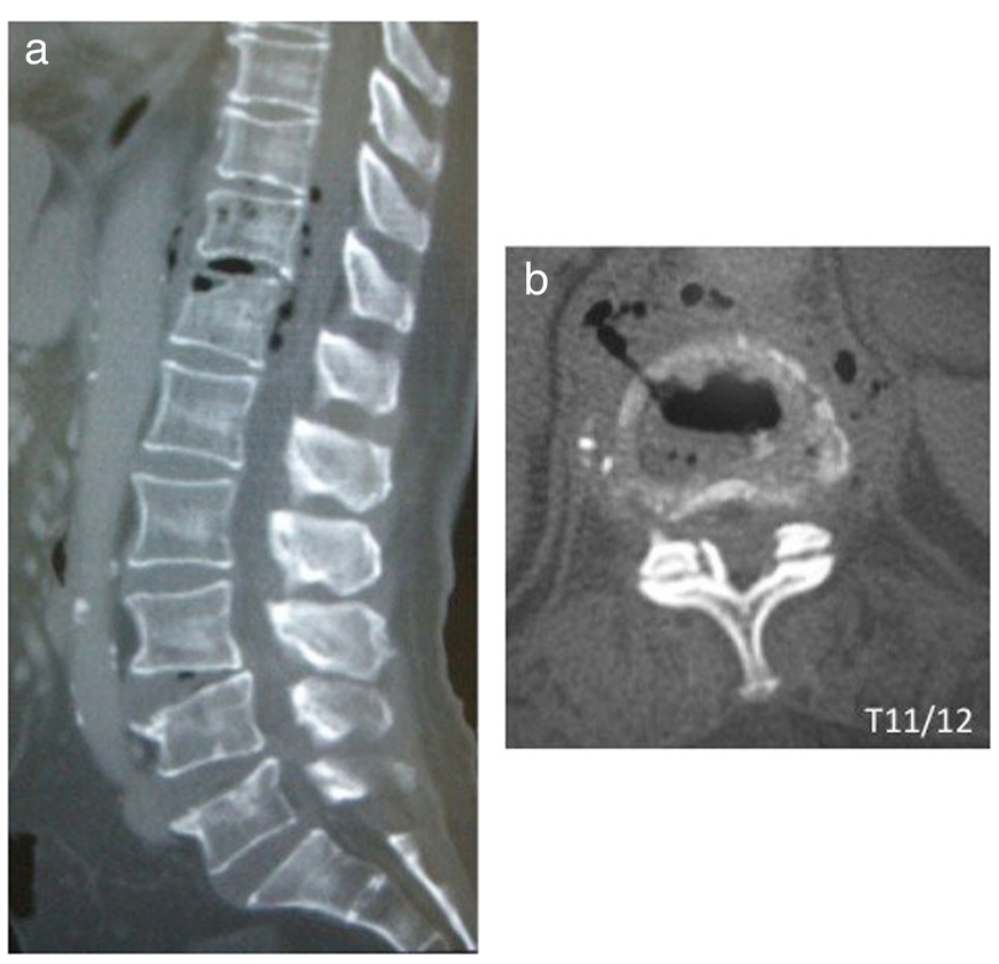

Figure 1 Computed tomography sagittal and axial images on initial admission. (a) Soft tissue swelling anterolaterally at intervertebral disc level T11/12 (b) and a gas-containing epidural abscess are seen. 

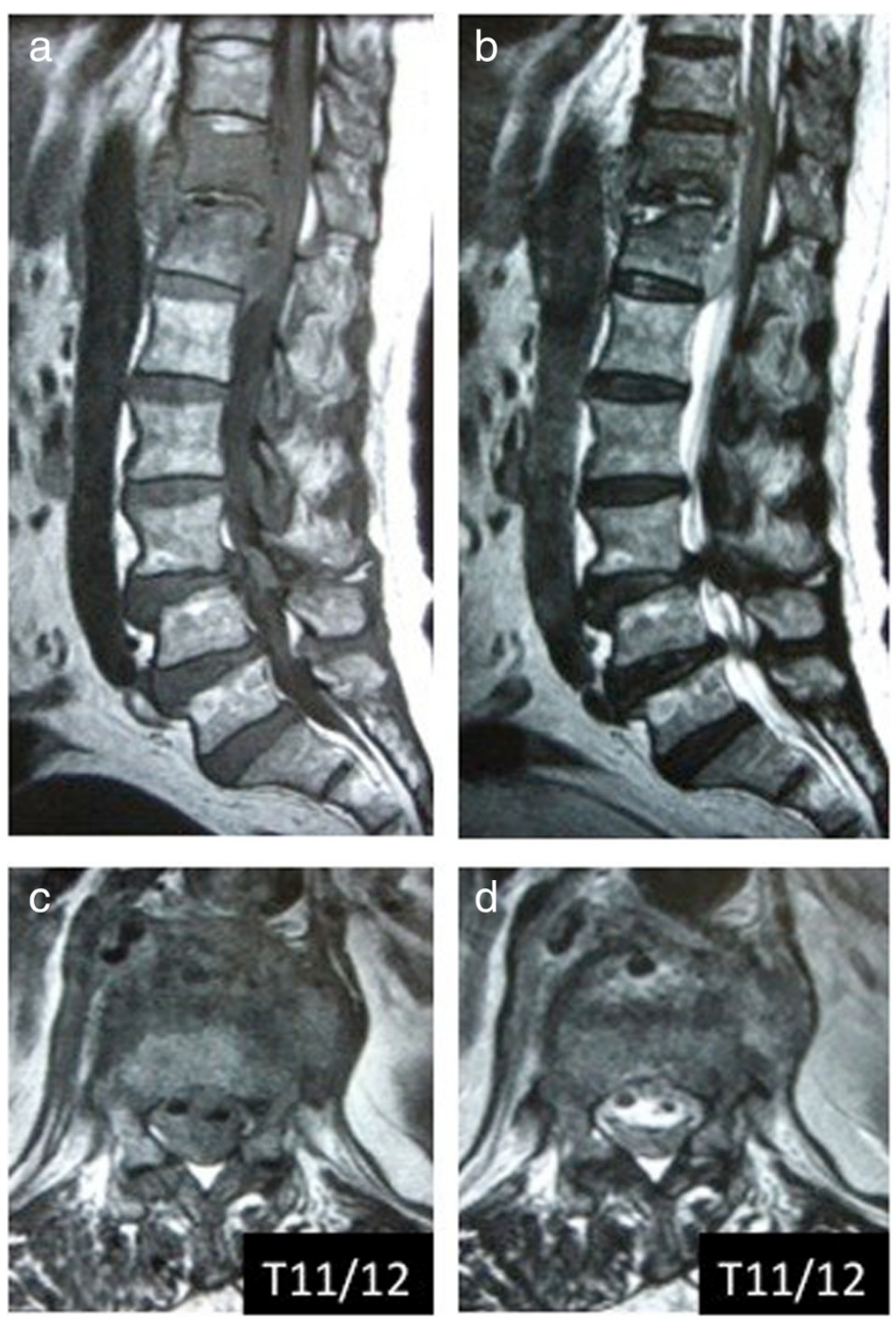

Figure 2 Magnetic resonance imaging sagittal and axial images on initial admission. Involvement of the T11/12 disc space and adjacent vertebral body with decreased signal intensity on the T1-weighted images (a and $\mathbf{c}$ ) and increased signal intensity on the T2-weighted images (b and $\mathbf{d}$ ) with a gas-containing epidural abscess are seen.

myonecrosis is caused by $C$. perfringens and Clostridium septicum in $60 \%$ and $30 \%$ of cases, respectively $[12,13]$. Clostridial species are commonly found in soil, dust, water, and the intestines of humans and various animals $[2,14,15]$. Without treatment, there is a $100 \%$ mortality rate within 24 hours of onset of systemic symptoms [14]. If properly treated, the overall mortality rate is 20 to $30 \%[2,3,16]$. The mortality rate is higher with trunk involvement $(50 \%)$ than with involvement of the extremities (24\%), which carries a better prognosis [2].

With respect to vertebral involvement, favorable outcomes after discitis caused by $C$. perfringens are obtained if the infection occurs only within the disc $[5,6,8,10]$. However, with surgical site infection (SSI) after lumbar spine surgery, one patient died, and one needed multiple operations $[4,7]$. The differences in outcomes may be related to the amount of ischemic tissue. Although the disc itself has a small amount of ischemic tissue and a favorable outcome, the amount of ischemic tissue with SSI is larger and is associated with a terrible outcome. The present case with discitis and an epidural abscess survived because the amount of ischemic tissue was small, and emergency debridement and antibiotic therapy were effective. 


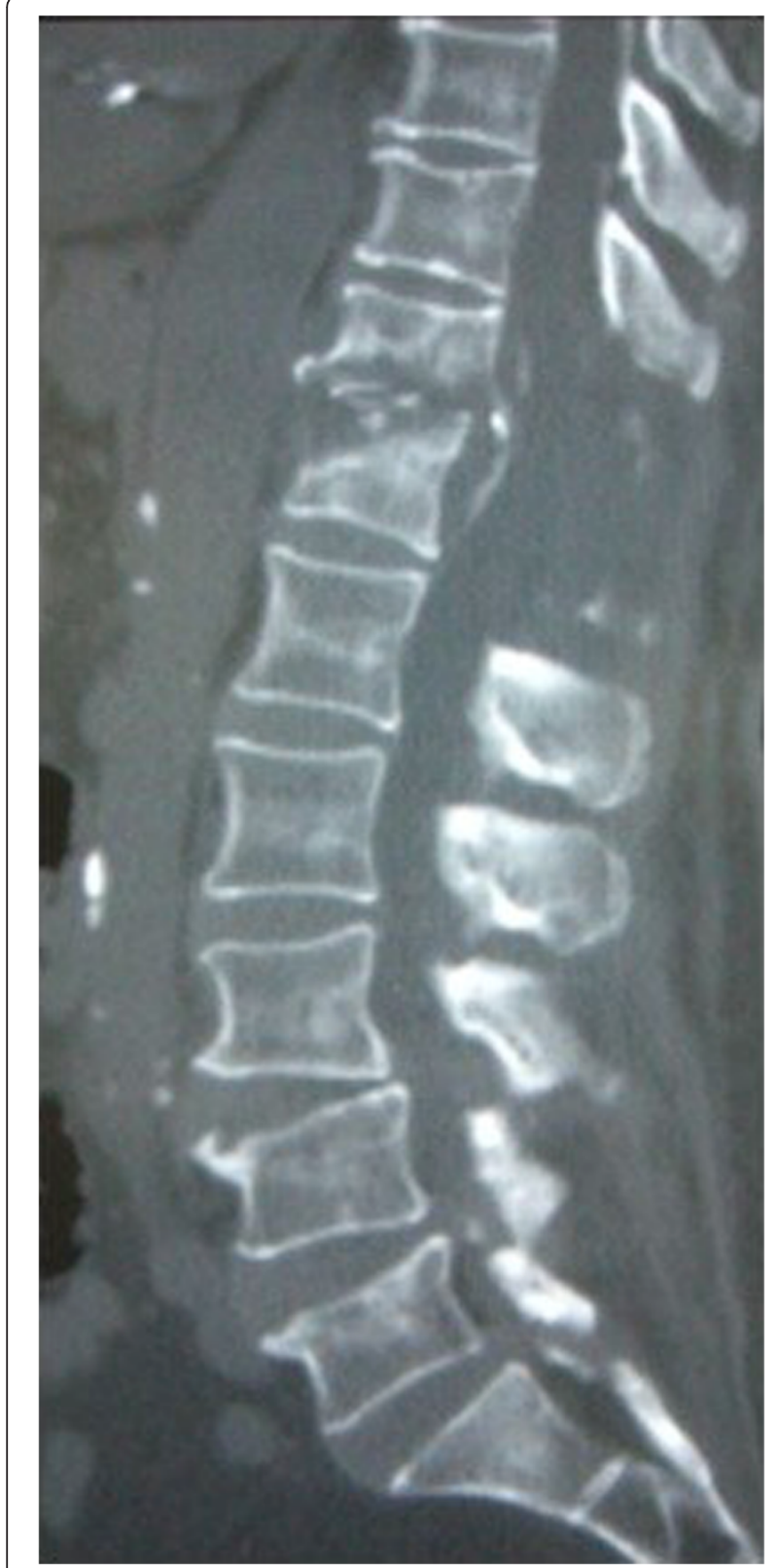

Figure 3 Computed tomography at 6 weeks after operation. T12 vertebral bone destruction is seen.

Fusion surgery is needed if instability remains [17]. Stabilization with instrumentation is a safe and effective treatment for pyogenic osteomyelitis [18-21]. In this case, even after her CRP level decreased, the patient could not sit because of her left lateral chest pain. On CT, vertebral bone destruction had appeared, and instability was considered the main reason for her lateral chest pain. We managed this patient with posterior fixation with instrumentation. She was treated successfully, and she was ambula-

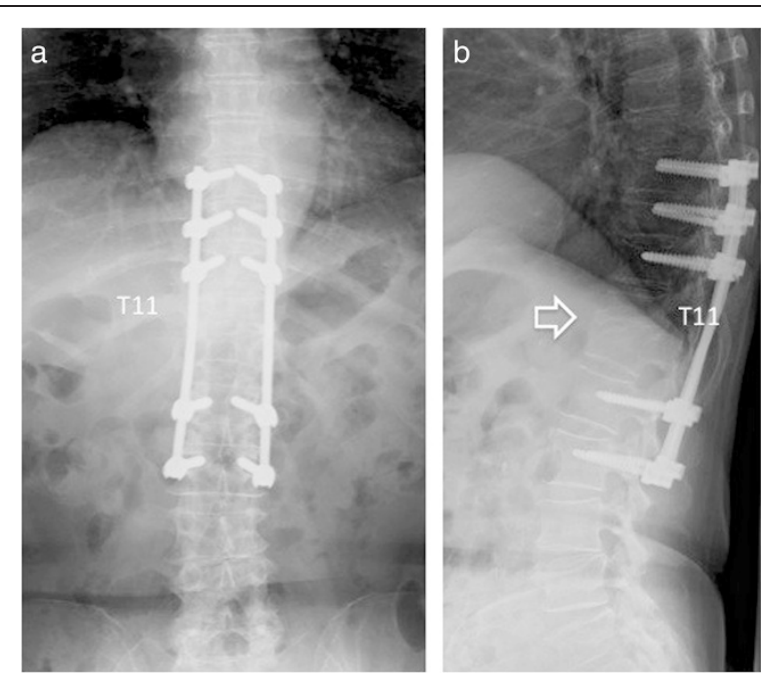

Figure 4 Plain radiograph at 18 months after operation. Complete union between the T11 and T12 vertebral bodies is seen. Open arrow shows bone bridge between T11 and T12 vertebra. (a) Anteroposterior radiograph, (b) Lateral radiograph.

tory and showed complete bone union on X-ray at 18-month follow-up.

\section{Conclusions}

A very rare case of vertebral osteomyelitis caused by gas gangrene that was successfully treated by immediate debridement and subsequent fusion surgery combined with antibiotics was described.

\section{Consent}

Written informed consent was obtained from the patient for publication of this case report and accompanying images. A copy of the written consent is available for review by the Editor-in-Chief of this journal.

\section{Abbreviations}

CRP: C-reactive protein; CT: computed tomography; SSI: surgical site infection; WBC: white blood cell count.

\section{Competing interests}

The authors declare that they have no competing interests.

\section{Authors' contributions}

Surgery was performed by TK. MA, TK, and NM were the major contributors in writing the manuscript. EA, TA, KK, and YS supervised the whole work. All authors read and approved the final manuscript.

\section{Acknowledgements}

The authors would like to thank Mamiko Kondo and Sachie Miura for their valuable assistance with the editing of this manuscript.

\section{Author details}

'Department of Orthopedic Surgery, Akita Kousei Medical Center, 1-1-1 lijima-Nishifukuro, Akita 011-0948, Japan. ²Department of Orthopedic Surgery, Akita University Graduate School of Medicine, 1-1-1 Hondo, Akita 010-8543, Japan. 
Received: 22 October 2014 Accepted: 4 March 2015

\section{Published online: 11 April 2015}

\section{References}

1. MacLennan JD. The histotoxic clostridial infections of man. Bacteriol Rev. $1962 ; 26: 177-276$

2. Caplan ES, Kluge RM. Gas gangrene: review of 34 cases. Arch Intern Med. 1976;136:788-91.

3. Darke SG, King AM, Slack WK. Gas gangrene and related infection: classification, clinical features and aetiology, management and mortality. A report of 88 cases. Br J Surg. 1977;64:104-12.

4. Bednar DA. Postoperative Clostridium perfringens lumbar discitis with septicemia: report of a case with survival. J Spinal Disord Tech. 2002;15:172-4.

5. Beguiristain JL, de Pablos J, Llombart R, Gómez A. Discitis due to Clostridium perfringens. Spine. 1986;11:170-2.

6. Caudron A, Grados F, Boubrit Y, Coullet JM, Merrien D, Domart Y. Discitis due to Clostridium perfringens. Joint Bone Spine. 2008;75:232-4.

7. Kristopaitis T, Jensen R, Gujrati M. Clostridium perfringens: a rare cause of postoperative spinal surgery meningitis. Surg Neurol. 1999;51:448-50.

8. Pate D, Katz A. Clostridia discitis: a case report. Arthritis Rheum. 1979:22:1039-40.

9. Thompson 3rd GR, Crawford GE. Pneumorachis caused by metastatic gas gangrene. Diagn Microbiol Infect Dis. 2009;63:108-10.

10. Torda AJ, Gottlieb T, Bradbury R. Pyogenic vertebral osteomyelitis: analysis of 20 cases and review. Clin Infect Dis. 1995;20:320-8.

11. Hart GB, Lamb RC, Strauss MB. Gas gangrene. J Trauma. 1983;23:991-1000.

12. Burke MP, Opeskin K. Nontraumatic clostridial myonecrosis. Am J Forensic Med Pathol. 1999:20:158-62.

13. Hiew CY, Silberstein M, Hennessy OF. Fatal Clostridium septicum myonecrosis. Australas Radiol. 1993;37:399-400.

14. Marty AT, Filler RM. Recovery from non-traumatic localised gas gangrene and clostridial septicaemia. Lancet. 1969;12:79-81.

15. Nandyala SV, Schwend RM. Prevalence of intraoperative tissue bacterial contamination in posterior pediatric spinal deformity surgery. Spine (Phila Pa 1976). 2013;15:E482-6.

16. Altemeier WA, Fullen WD. Prevention and treatment of gas gangrene. JAMA. 1971:217:806-13.

17. Busche M, Bastian L, Riedemann NC, Brachvogel P, Rosenthal H, Krettek C. Complete osteolysis of the dens with atlantoaxial luxation caused by infection with Staphylococcus aureus: a case report and review of the literature. Spine (Phila Pa 1976). 2005;30:E369-74.

18. Abe E, Yan K, Okada K. Pyogenic vertebral osteomyelitis presenting as single spinal compression fracture: a case report and review of the literature. Spinal Cord. 2000;38:639-44.

19. Menon VK, Kumar KM, Al GK. One-stage biopsy, debridement, reconstruction, and stabilization of pyogenic vertebral osteomyelitis. Global Spine J. 2014:4:93-100

20. Mohamed AS, Yoo J, Hart R, Ragel BT, Hiratzka J, Hamilton DK, et al. Posterior fixation without debridement for vertebral body osteomyelitis and discitis. Neurosurg Focus. 2014;37:E6.

21. Suchomel P, Buchvald P, Barsa P, Lukas R, Soukup T. Pyogenic osteomyelitis of the odontoid process: single stage decompression and fusion. Spine (Phila Pa 1976). 2003;28:E239-44.

\section{Submit your next manuscript to BioMed Central and take full advantage of:}

- Convenient online submission

- Thorough peer review

- No space constraints or color figure charges

- Immediate publication on acceptance

- Inclusion in PubMed, CAS, Scopus and Google Scholar

- Research which is freely available for redistribution 